\title{
Beobachtungen von Sternschnuppen 1892 Nov. 19-27
}

\author{
auf der Sternwarte der k. k. böhmischen (czechischen) Universität in Prag.
}

Betreffend den Sternschnuppenfall Nov. 23 erlaube ich mir Folgendes mitzutheilen:

1892 Nov. $198^{\mathrm{h}}-9^{\mathrm{h}}$ Abends helle Sternschnuppen.

Nov. 23 nach 10 Uhr Abends hăufige Sternschnuppen, jede Minute wenigstens eine; um 16 Uhr häufige Sternschnuppen von kurzen Bahnstrecken mit ausgepragter Radiation aus Andromeda und auch Cassiopeia.

Nov. 24 Abends $8^{\text {h }}$ sehr wenige, fruh $16^{\text {b }}$ keine Sternschnuppen. Ganz heiter. Luft gut $u$. durchsichtig.

Nov. 25 frlih $17^{\mathrm{h}}-17^{3} /^{\mathrm{b}}$ keine Sternschnuppen. Ganz heiter.

Nov. 26 früh $17^{\text {h }}-17^{1} /^{\text {h }}$ keine Sternschnuppen. Ganz heiter.

Nov. 27 frtih $15^{3} /^{\mathrm{h}}-161 /^{\mathrm{h}}$ sehr wenige Sternschnuppen. Heiter.

Nov. 20 bis 22 schlechtes Wetter.

Beobachter : Gruss und Dr. Ldska.

Prag VII 80, 1892 Dec. 17.

G. Gruss.

\section{Osservazioni di Stelle Variabili a lungo periodo.}

$$
\text { (v. A. N. } 3036 \text {.) }
$$

Di Francesco Porro.

\section{$1855 \mathrm{R}$ Aurigae.}

Osservata sette volte fra il 4 Dicembre 189 I e il 27 Gennajo 1892 . Nell' intervallo scese gradatamente dalla settima alla nona grandezza. Color rosso intenso, notato specialmente il 21 Dicembre.

\section{S Persei.}

Tre osservazioni di questa stella confermano pienamente la sua costanza intorno ad un massimo $7 \% 7$, annunziata dal Prof. Safarik nel No. 26r dell' Astronomical Journal. La stella si mantenne sempre tre gradazioni maggiore della $c$ del catalogo di Pickering, fra il Dicembre 8 e il Gennajo 29.

\section{T Persei.}

Osservata insieme alla precedente in Dicembre $189 \mathrm{I}$ e Gennajo r892. Aumentò dalla grandezza 9 alla 8.5.

\section{S Cassiopeize.}

Sei osservazioni dal 5 Dicembre 189 r al 29 Gennajo 1892. Alla prima la stella era già inferiore di circa mezza grandezza alla $m$ di Pickering, e decrebbe lentamente alle successive, scendendo al disotto dell' undecima grandezza. Fra il 17 e il 21 Dicembre pare sia avvenuta una grande variazione (quasi una grandezza) nello splendore relativo delle due stelle di comparazione $m$ ed $o$.

\section{R Lyncis.}

Osservata in cinque notti fra il 5 Dicembre 1891 e il 2 Gennajo 1892 . Si mantenne costante intorno alla decima grandezza.

\section{T Cassiopeiae.}

L' 8 Dicembre 1891 questa stella non superava la grandezza 7 , essendo compresa fra la $c$ e la $d$ di Pickering (più vicino a questa che a quella).
7769 S Cephei.

Verso la decima grandezza il 22 ed il 28 Dicembre 189 r. Molto rossa.

$$
2100 \text { U Orionis. }
$$

Al 4 Dicembre 1891 ho ripreso le osservazioni di questa stella, essendone lo splendore di poco superiore alla decima grandezza nella scala di Argelander. Ia curva, costruita sopra 19 osservazioni, differisce da quelle degli anni precedenti, presentando un aumento di luce meno rapido ed un massimo più sicuro a stabilirsi, intorno al 12 Febbrajo 1892. La massima grandezza fu, come al solito, di poco superiore a quella delle due stelle di comparazione $c=\mathrm{BD}$. +19.1126 e $d=\mathrm{BD}$. +20\%1110, e quindi certamente compresa fra 5.5 e 6.0. Giova notare che $d$ si mantenne prevalentemente maggiore di $c$, al contrario di quanto avvenne negli anni scorsi e di quanto risulta dalle misure fotometriche di Cambridge e di Potsdam, $e$ invece d'accordo con BD. Come negli anni scorsi, la diminuzione di luce dopo il massimo è assai meno rapida che l' aumento prima di questo: non è prima del 17 Aprile che la stella raggiunge $l^{\prime}$ ottava grandezza, gia toccata il 3 Gennajo nella fase ascendente.

Le mie osservazioni dell' ultimo triennio lasciano forti motivi di credere che il periodo fra due massimi consecutivi di questa interessante variabile non sia costante, ma vada diminuendo di anno in anno. Infatti gli ultimi tre massimi da me determinati sono:

$$
\begin{array}{llr}
1890 & \text { Gennajo } 26 \\
1891 & \text { Febbrajo } & 5 \\
1892 & \text { Febbrajo } 12
\end{array}
$$

e, per l' assoluta equivalenza delle condizioni d' osservazioni, si possono ritenere utilmente comparabili fra loro.

Le discrepanze notevoli fra $i$ diversi osservatori che negli ultimi anni si sono occupati di questa variabile (spiegabilissime col fatto della lunga durata del massimo e col 